\title{
Gait Identification Using Inertial Sensors for Control of Adaptive Knee Orthosis Device
}

\author{
Yaojung Shiao, Ting-Yue Chang, and Chien-Hung Lai ${ }^{1,2^{*}}$ \\ Department of Vehicle Engineering, National Taipei University of Technology, \\ 1, Sec. 3, Chung-hsiao E. Road, Taipei 106, Taiwan \\ ${ }^{1}$ Department of Physical Medicine and Rehabilitation, School of Medicine, College of Medicine, \\ Taipei Medical University, $250 \mathrm{Wu}$-Xing Street, Taipei 110, Taiwan \\ ${ }^{2}$ Department of Physical Medicine and Rehabilitation, Taipei Medical University Hospital, \\ $252 \mathrm{Wu}$-Xing Street, Taipei 110, Taiwan
}

(Received May 2, 2017; accepted October 11, 2017)

Keywords: gait, gait control, gait analysis, inertial sensor

People with gait movement problems usually need the assistance of a knee orthosis device. A well-designed active or semiactive knee orthosis (SAKO) device can provide proper resistance torque to support an injured knee. Thus, gait phase or gait movement must be identified precisely for the control algorithm to determine the correct output torque command. In this research, we investigate the application of an inertial sensor for different locations on a shoe. Three inertial sensors are attached to the heel, side, and instep of one shoe for testing. Multiaxis acceleration signals from these three sensors are measured simultaneously for several gait cycles. The test results show that the shoe lateral sensor can provide proper signals of gait acceleration for the realtime gait control of the knee orthosis device.

\section{Introduction}

People with gait movement problems usually require the assistance of a knee orthosis device. A well-designed active or semiactive knee orthosis (SAKO) device can provide proper resistance torque to support an injured knee. Currently, the conventional knee orthosis (CKO) device is very commonly used because of its simple structure and low cost. A CKO device helps a user maintain stance stability and free knee swing. However, a CKO device cannot enable a user to have a natural gait.

Active knee orthosis (AKO) device has been developed to help a user have natural gait movement. An AKO device usually combines a knee orthosis frame with an active actuator, sensors, and a controller. An active actuator can lock or force knee movement. With a welldesigned control algorithm, an AKO device can enable a user to walk more naturally. Beyl et al. ${ }^{(1)}$ developed an adaptive knee orthosis with an electric motor. However, an AKO device usually has problems of heavy mass, high power consumption, complex structure, and high cost. Therefore, the AKO device is mostly used by persons with serious gait problems.

Compared with the AKO device, a SAKO device has a resistance device instead of the heavy active electric motor. This resistance device can provide a variable resistance torque to provide

*Corresponding author: e-mail: chlai@tmu.edu.tw

http://dx.doi.org/10.18494/SAM.2017.1739 
a user with assistance in stance stability and leg swing. The SAKO device has a lighter orthosis frame, a lower power consumption, and a lower cost than the AKO device. Weinberg et al. ${ }^{(2)}$ reported a SAKO device with an electrorheological (ER) resistance device, which produces a 12.6 $\mathrm{N} \cdot \mathrm{m}$ maximum torque to adaptively restrict knee movement. However, the high mass and high ER voltage limit its practical applications. Another SAKO device developed by Shiao et al. ${ }^{(3)}$ adopts a newly designed magnetorheological (MR) resistance device. The proposed MR device has a multilayer structure and a high torque-to-volume ratio, so the SAKO device can provide a high torque, a low mass, and a small volume. An MR SAKO device is shown in Fig. 1.

Gait control by the AKO or SAKO device requires precise information about the gait phase or gait movement. Thus, the motor in the AKO device or the MR device in the SAKO device can output the correct torque command to restrict or to free knee movement for gait control. The human gait cycle was defined by the Rancho Los Amigos gait analysis committee. The gait movement is divided into eight functional phases: initial heel contact, loading response, midstance, terminal stance, preswing, initial swing, midswing, and terminal swing phases. ${ }^{(4)}$ For each phase, each sole will experience pressure acting on different sole areas. Therefore, the sole pressure in each phase characterizes a pressure distribution pattern, and these patterns can be used to identify the sole phase or sole movement.

\section{Gait Measurement}

In gait control, the actuator (motor or resistance device) must provide maximal torque to prevent the knee from bending at the stance phase. This torque is gradually decreased at the toe-off phase to let the leg swing freely. When a gait reaches its heel strike phase, this torque will increase gradually to a maximum value to lock the knee at the stance phase.

Gait measurement is very important for the control of the AKO and SAKO devices. In the rehabilitation of a knee or an ankle, only some key gait measurement information is sufficient to perform gait analysis. However, gait analysis for active or semiactive gait control requires complete and accurate gait measurement. In gait control, gait measurement is fed into a control algorithm to estimate the movements of the knees, ankles, and feet at any moment. Then, each gait step can be identified for gait control in order to determine the output torque command.

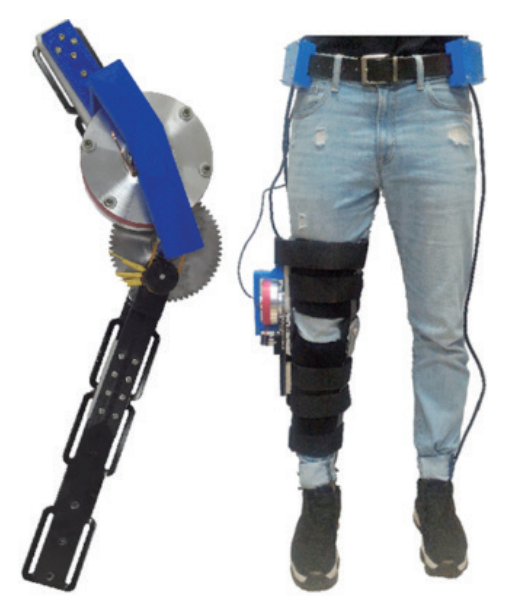

Fig. 1. (Color online) SAKO device with a magnetorheological device. ${ }^{(3)}$ 
Currently, several methods are applied by researchers to measure or estimate the information of gait movement. This information includes positions and moving velocities and accelerations of the knee, thigh, shin, foot, or ankle. The measured data is adopted to estimate the gait movement. Then, gait control will actuate the motor or resistance device to enable smooth knee movement or ankle movement in accordance with the gait estimation. Thus, the gait estimation should be relatively precise in this process. This means that one set of gait measurements must enable the determination of a unique gait movement.

Because of the rapid advancement of sensor technology, there are several sensing methods that can be adopted to collect information about the movements of the knee and ankle for gait analysis. One conventional way is to determine the positions of the knee, thigh or shin optically, similar to motion capture technology. However, the equipment setup is complicated and the cost is relatively high. A mechanical potentiometer and a tachometer are also fixed to the thigh and shin for gait measurement. Unfortunately, these sensors have problems in precise measurement because the human leg is not an ideal rigid body with a fixed rotating center in the knee.

The sole pressure distribution is another method of gait measurement. A complete gait cycle is composed of a stance phase and a swing phase, and usually starts from a right heel strike and ends at the next right heel strike. Figure 2 shows a complete gait cycle. If the pattern of the pressure distribution of both soles is unique for each action in a gait cycle, then the sole pressure distribution is a good index for the estimation of gait movement.

\subsection{Force sensor}

To identify the position in a gait cycle for each moment, the sole pressure distribution is commonly adopted by researchers and in the industry. Various devices have been developed to measure the sole pressure distribution. For years, the floor reaction force gauge has been a common and useful sensing tool. It provides a full and clear pressure distribution of both soles for each moving moment. However, its large dimensions, gait space limitation, and low portability are its critical drawbacks for application in controlled knee orthosis.

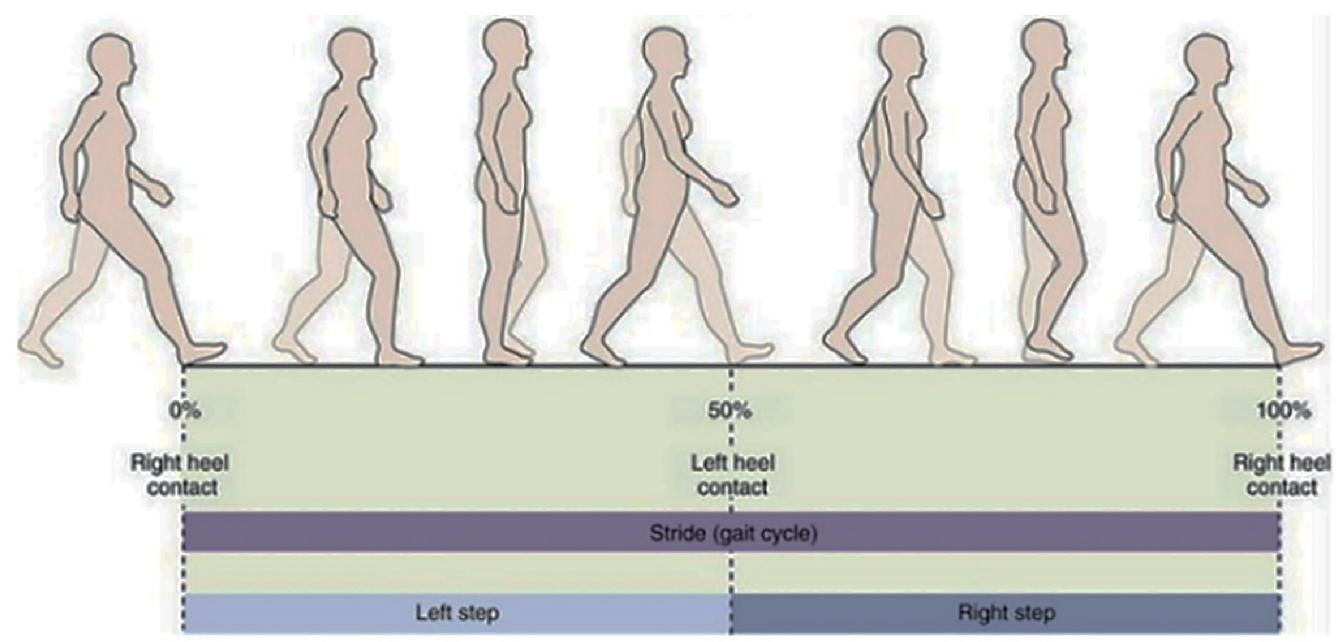

Fig. 2. (Color online) A complete gait cycle. ${ }^{(5)}$ 
The insole-type measurement device has also been used in gait analysis in recent years. ${ }^{(3,6)}$ During gait movement, the sole has different pressure distributions. Each gait is characterized by the pattern of the sole pressure distribution. Figure 3 shows the sole pressure distribution during a gait cycle. ${ }^{(7)}$ At the beginning of the gait cycle, a high pressure acts on the heel area, while the major pressure transfers to the toe at the midstance period. There is no pressure on the sole during the swing phase.

Air-pressure sensors were applied in sole pressure measurement by Kong. ${ }^{(7)}$ There are four air bladders located under one sole to measure the individual applied pressure. Such an airtype measurement provides more comfort and a larger detection range than a load-cell-type measurement. However, its large size makes it unsuitable as a wearable measurement device for real-time gait control.

The insole-type slim load cell is also adopted in gait control to measure sole pressure. The force sensor is small, thin, and light. Usually, two to seven force sensors are used together to measure the sole pressure. According to the observation of the sole pressure distributions in Fig. 3, the highest sole pressure occurs at the areas of the medial heel, the second metatarsal head, and the great toe. An insole with slim load sensors is shown in Fig. 4. Three load sensors are located in the insole.

The measured sole forces are shown in Fig. 4. Sensor $\mathrm{C}$ measures a high sole pressure after a heel strike, and sensors A and B then detect high pressure signals at midstance. From the measured

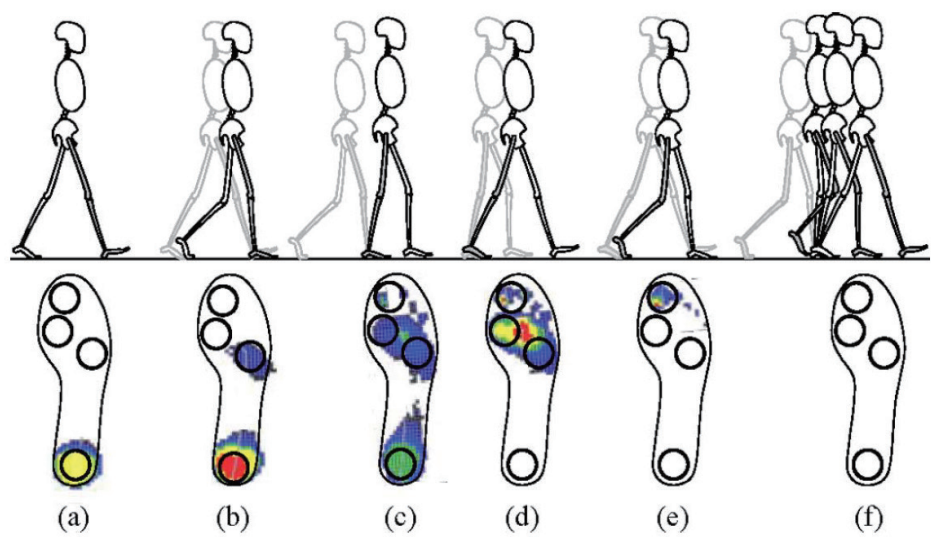

Fig. 3. (Color online) Complete sole pressure distributions during a gait cycle. ${ }^{(7)}$
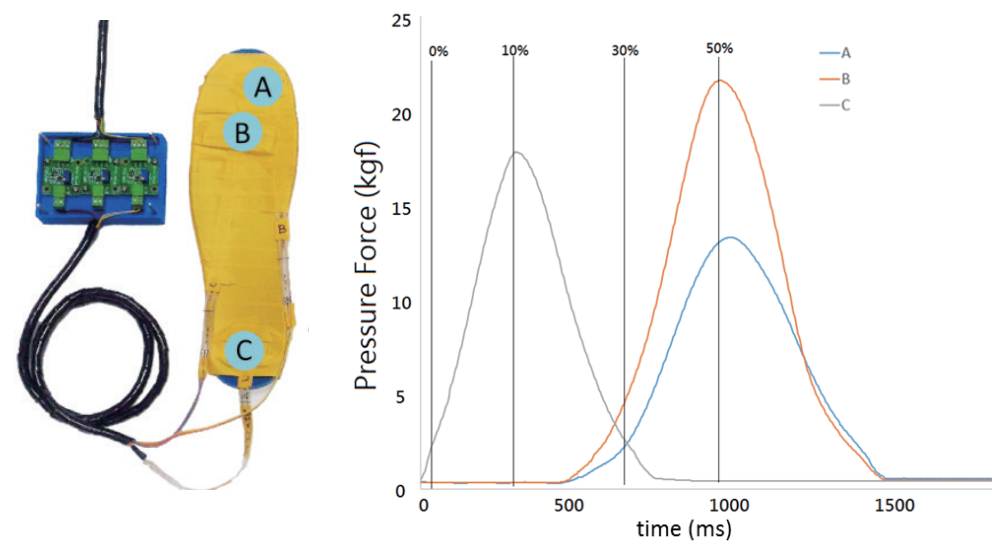

Fig. 4. (Color online) Insole with slim load sensors and measured sole forces. 
profiles of the medial heel (sensor C) and second metatarsal head (sensor B), each gait movement can be roughly characterized. However, this type of gait measurement still has a problem in its application to real-time gait control. The wire connection is not sufficiently durable for this use, and also obstructs the user from walking. Besides, it is inconvenient and uncomfortable for the user to wear the special insole every day. Each force sensor must also be installed precisely at a specific sole location. Another easy and convenient measurement method is needed for gait control.

\subsection{Inertial sensor}

Other than the insole pressure distribution, gait movement can also be characterized by swing accelerations and angular velocities. Because of the advanced electronic sensor technology, many multiaxis inertial sensors are available for teaching, research, and industrial applications. A sixaxis sensor is very common for engineering applications nowadays. It is light, small, and easy to install. In this research, we use the six-axis inertial sensor MPU 6050 to measure the foot swing acceleration during a gait cycle. The MPU can measure three-axis acceleration and three-axis angular velocity. Because of its slim size, it can be attached to the foot or shoes of a user. This makes the MPU very suitable and convenient for applications in gait control and the rehabilitation exoskeleton of legs.

\section{Gait Measurement by Inertial Sensor}

Recently, inertial sensors are gradually being used in rehabilitation. Aoike et al. ${ }^{(8)}$ attached an MPU 6050 inertial sensor at the shoe heel and obtained the acceleration signals during gait movement. In their research, the $z$-axis is opposite the direction of movement and the $y$-axis is the vertical direction. According to their measurement results (Fig. 5), a heel strike is mainly identified by a positive peak in the $z$-axis acceleration, while the toe off is identified by a vertical $y$-axis signal. However, the $y$-axis signal has two positive peaks during one gait cycle, and these two peaks can be confused by the gait control algorithm in the control of the motor and resistance device.
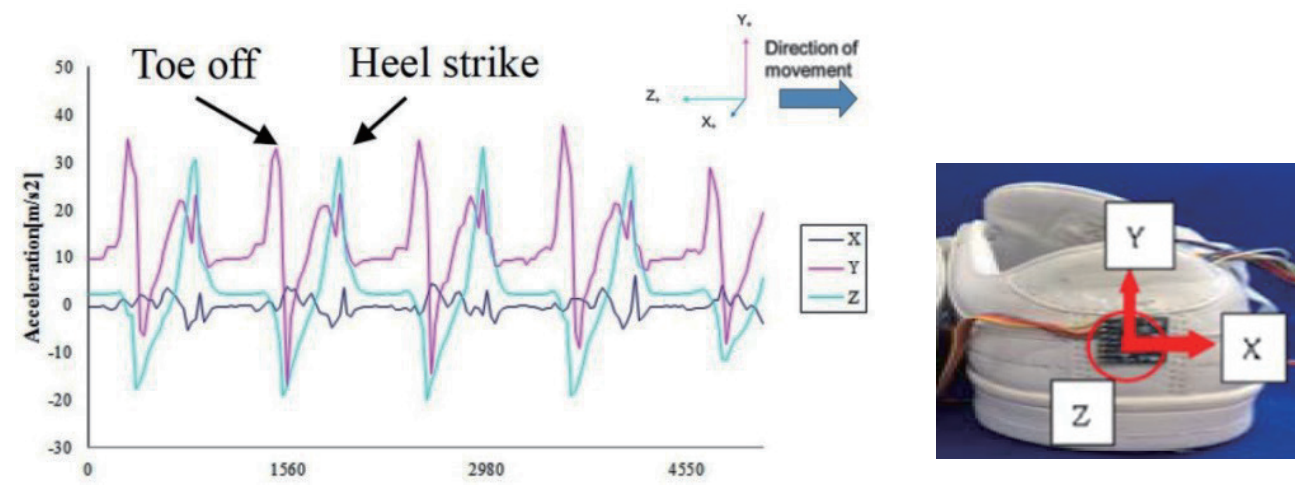

Fig. 5. (Color online) Results of measurement using MPU 6050 at shoe heel. ${ }^{(8)}$ 


\subsection{Sensor installation location}

In this research, we investigate the different installation locations for the MPU inertial sensor to determine an optimal location for sensor installation. Considering the convenience of a user undergoing rehabilitation, we test three locations: shoe heel, shoe lateral, and shoe instep (Fig. 6). For each MPU installation, the $y$-axis is in the vertical direction for the shoe heel and shoe lateral, and in the backward moving direction for the shoe instep.

\subsection{Sensor measurement}

Three MPU inertial sensors are attached to the specific locations simultaneously to measure gait accelerations in three axes. From the simultaneous acceleration data, it becomes easy to decide which location is better for MPU installation and gait measurement.

For the MPU sensor on the shoe heel (Fig. 7), the $x$-axis acceleration is the lateral swing acceleration and can be neglected because it is unrelated to gait movement. The $y$-axis acceleration is in the vertical direction and has a value of 9.81 at the stance phase. Because the sensor location is near the heel, the sensor detects a strong reaction in the heal-off and heel-strike phases. The $y$-axis acceleration in Fig. 7 shows its first peak at the heel off and its second peak just before the

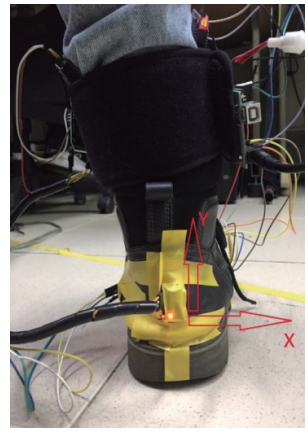

(a)

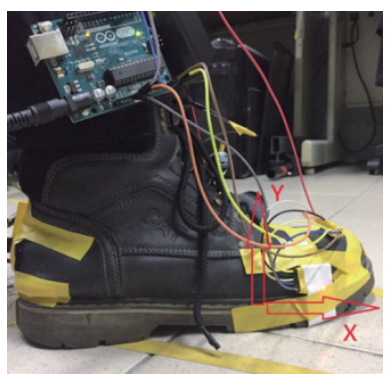

(b)

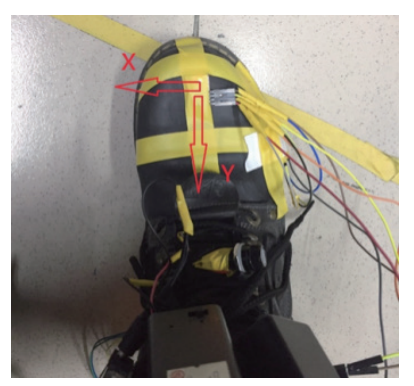

(c)

Fig. 6. (Color online) Three locations of installation of MPU inertial sensors.

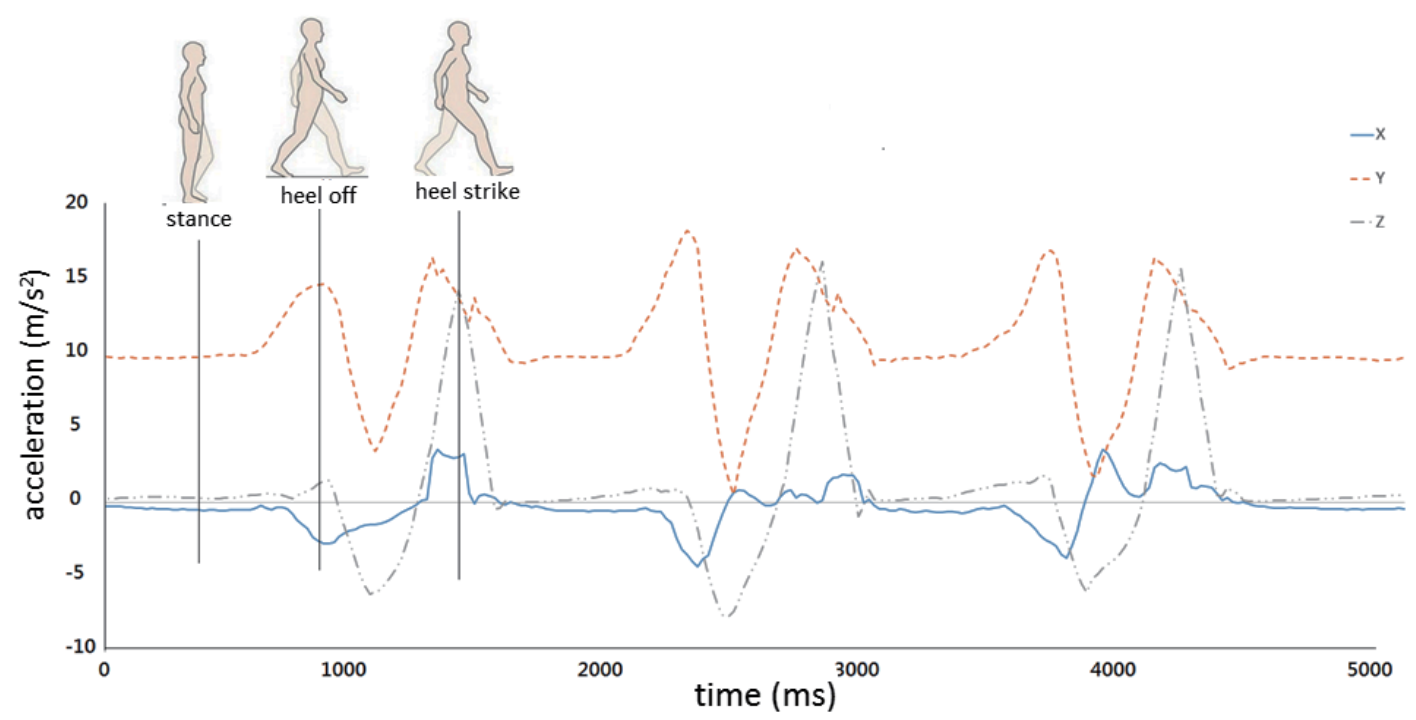

Fig. 7. (Color online) Accelerations with sensor at shoe heel. 
heel strike. Meanwhile, the $z$-acceleration reaches its peak at the heel strike. Thus, the heel-off phase can be identified by the first peak of the $y$-acceleration, and the moment of heel strike can be identified from the positive peak of the $z$-acceleration.

If the MPU sensor is attached to the shoe side (Fig. 8), the $z$-acceleration is the lateral swing acceleration and it has no characteristic in forward movement. The $y$-axis is in the vertical direction and its value is strongly affected by the actions of the heel off and heel strike. In Fig. 8, a positive peak of the $y$-acceleration can be easily observed at the moment of heel off. However, the $y$-acceleration has no significant characteristic to identify the moment of heel strike because the sensor is far from the heel. Meanwhile, a large negative peak in the $x$-axis occurs at the moment of heel strike. Thus, it is easy to explicitly identify the timing of heel off by the first $y$-acceleration peak, and to identify the heel strike by following the large negative $z$-acceleration peak.

When an MPU sensor is installed at the shoe instep (Fig. 9), the $x$-axis acceleration is the lateral swing acceleration. This $x$-axis acceleration is a small value and has a weak relationship with the forward gait movement. The vertical acceleration is measured in the $z$-axis direction. Thus, in the stance phase, the $z$-axis acceleration has a value of 9.81 while the $x$ - and $y$-axes have zero values

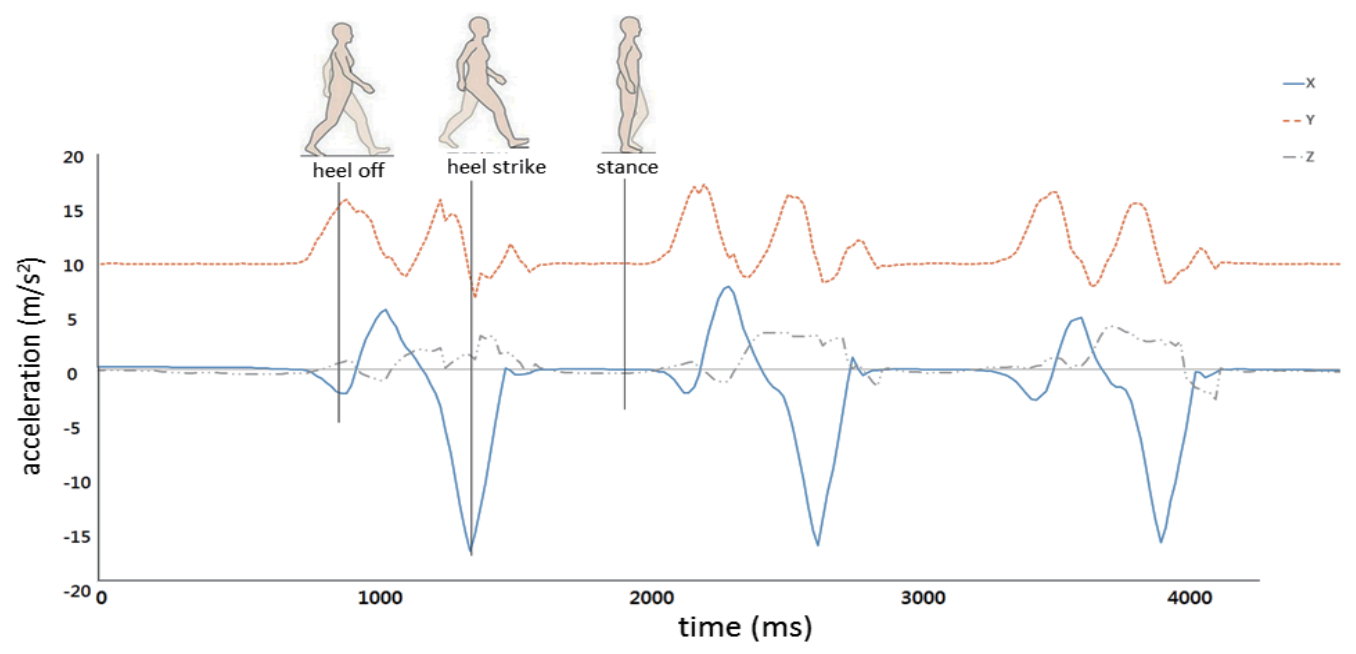

Fig. 8. (Color online) Accelerations with sensor at shoe side.

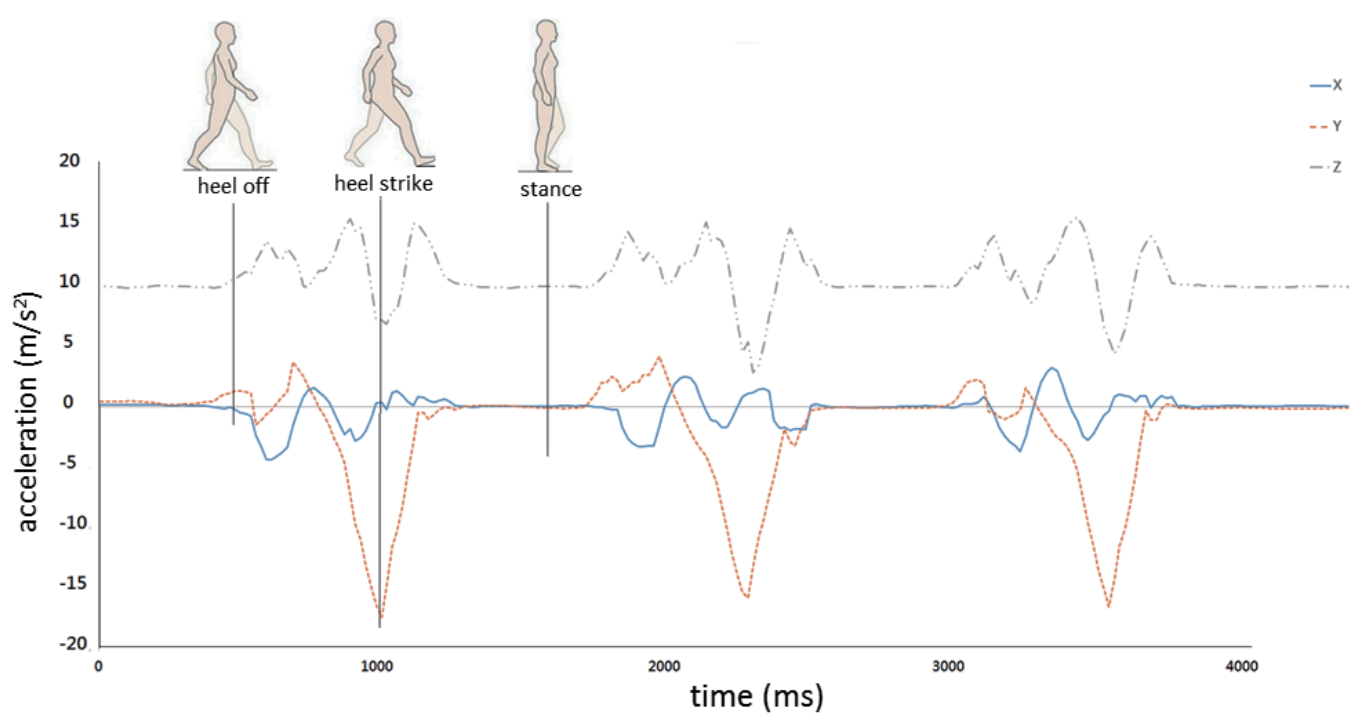

Fig. 9. (Color online) Accelerations with sensor at shoe instep. 
for their acceleration. The measured three-axis accelerations are shown in Fig. 9. At the heeloff phase, there is a small positive acceleration in the $y$-axis. During the swing phase, the sensor detects a negative $y$-axis acceleration, and reaches a peak value at the moment of heel strike. Thus, this large negative peak along the $y$-axis direction becomes a significant characteristic of the swing phase from heel off to heel strike. However, there is no significant characteristic to identify the moment of heel off.

\subsection{Gait analysis for control}

Gait measurement using the MPU inertial sensor can reduce the complexity of sensor installation and wiring. Moreover, it is also convenient to install and use daily for real-time gait control. Tests with all the sensors at different installation locations has been performed to compare the signal sensitivity with the forward gait movement. As mentioned earlier, the controlled actuator output torque gradually decreases from the timing of heel off, remains zero for free swing, and increases to its maximum from the timing of heel strike. Thus, the key points in gait identification are the timings of heel off, toe off, and heel strike. Figures 7-9 show the three-axis accelerations in three different locations.

For the case of the sensor at the shoe instep (Fig. 9), it is clear that the timing of heel off is difficult to determine from either the $y$ - or $z$-acceleration. Thus, the shoe-instep location is not appropriate for gait measurement for gait control. Figures 7 and 8 show that the heel off can be easily characterized by the $y$-acceleration, and the timing of the heel strike can be well identified by the $z$-acceleration measured at the shoe heel or by the $x$-acceleration measured at the shoe side. However, the up-and-down heel movements produce some similar signal profiles during a gait cycle in the shoe heel case. It causes difficulty in correctly identifying the timing of heel off from the signal profiles. This nonunique signal-to-action matching can confuse a gait control algorithm and cause an incorrect output torque command to be sent to an actuator. Then, the knee could be incorrectly locked or set free, consequently causing the user to fall down seriously. Therefore, it is concluded that the shoe side position is the optimal sensor location for obtaining appropriate acceleration signals to identify well the gait movement for real-time gait control.

\section{Conclusions}

The identification of gait phase and movement is very important for real-time gait control through the use of active or semiactive actuators of a knee orthosis device. To provide precise torque from a control actuator, each gait phase should be identified correctly and explicitly, especially the timings of heel off, toe off, and heel strike. In this research, we investigated several gait sensing methods for real-time gait control using an insole-type force sensor and threeaxis inertial sensors. For the adoption of the inertial sensor, three different shoe locations were investigated to determine good locations for sensor installation. Three inertial sensors detected the three-axis accelerations simultaneously for several gait cycles. The results show that gait movement can be well identified by the method in which the inertial sensor is attached to the shoe side. 


\section{Acknowledgments}

The authors would like to thank the NTUT-TMU Joint Research Program for the funding support (Project No.: NTUT-TMU-103-04).

\section{References}

1 P. Beyl, J. Naudet, R. Van Ham, and D. Lefeber: Proc. 2007 IEEE 10th Int. Conf. Rehabilitation Robotics (2007) p. 100.

2 B. Weinberg, J. Nikitczuk, S. Patel, B. Patritti, C. Mavroidis, P. Bonato, and P. Canavan: Proc. 2007 IEEE Int. Conf. Robotics and Automation (2007) p. 4126.

3 Y. Shiao, C.-H. Lai, and N.-C. Kao: Proc. 2016 Int. Multi-Conf. Engineering and Technology Innovation (2016).

4 J. Perry: Gait Analysis (Slack, Inc., New Jersey, 1992).

5 G. Simoneau: Kinesiology of Walking: Kinesiology of the Musculoskeletal System (Mosby, St. Louis, 2010) p. 627.

6 W. Samson, S. Sanchez, P. Salvia, S. V. S. Jan, and V. Feipel: Gait Posture 40 (2014) 420.

7 K. Kong: IEEE Trans. Rob. 14 (2009) 358.

8 K. Aoike, K. Nagamune, K. Takayama, R. Kuroda, and M. Kurosaka: Proc. 2016 IEEE Int. Conf. Systems, Man, and Cybernetics (2016).

\section{About the Authors}

Yaojung Shiao received his B.S. degree from National Cheng-Kung University, Taiwan, in 1986 and his M.S. and Ph.D. degrees from the University of Wisconsin-Madison, U.S.A., in 1990 and 1995, respectively. From 1997 to 2001, he was an associate professor at National Pingtung University of Science and Technology, Taiwan. Since 2001, he has been an associate and full professor at National Taipei University of Technology, Taiwan. His research interests are in magnetorheological applications, advanced electric engine valvetrain, intelligent control, and gait control.

Chien-Hung Lai received his M.D. degree from Kaohsiung Medical University, Taiwan, in 1993 and his Ph.D. degree from the Department of Biomedical Engineering, Chung Yuan Christian University, Taiwan, in 2010. From 2011 to 2014, he was an assistant professor at Taipei Medical University, Taiwan. Since 2014, he has been an associate professor at Taipei Medical University. His research interests are in bioengineering, sensors, balance, fall prevention, and gait analysis.

Ting-Yue Chang received his B.S. degree from National Chin-Yi University of Technology, Taiwan, in 2015, and his M.S. degree from National Taipei University of Technology, Taiwan, in 2017. 